\title{
Distance Features for General Game Playing Agents
}

\author{
Daniel Michulke ${ }^{1}$, Stephan Schiffel ${ }^{2}$ \\ ${ }^{1}$ Department of Computer Science, Dresden University of Technology, Dresden, Germany \\ ${ }^{2}$ School of Computer Science, Reykjavík University, Reykjavík, Iceland \\ daniel.michulke@mailbox.tu-dresden.de,stephans@ru.is
}

Keywords: General Game Playing, Feature Construction, Heuristic Search

\begin{abstract}
General Game Playing (GGP) is concerned with the development of programs that are able to play previously unknown games well. The main problem such a player is faced with is to come up with a good heuristic evaluation function automatically. Part of these heuristics are distance measures used to estimate, e.g., the distance of a pawn towards the promotion rank. However, current distance heuristics in GGP are based on too specific detection patterns as well as expensive internal simulations, they are limited to the scope of totally ordered domains and/or they apply a uniform Manhattan distance heuristics regardless of the move pattern of the object involved.

In this paper we describe a method to automatically construct distance measures by analyzing the game rules. The presented method is an improvement to all previously presented distance estimation methods, because it is not limited to specific structures, such as, Cartesian game boards. Furthermore, the constructed distance measures are admissible.

We demonstrate how to use the distance measures in an evaluation function of a general game player and show the effectiveness of our approach by comparing with a state-of-the-art player.
\end{abstract}

\section{Introduction}

While in classical game playing, human experts encode their knowledge into features and parameters of evaluation functions (e.g., weights), the goal of General Game Playing is to develop programs that are able to autonomously derive a good evaluation function for a game given only the rules of the game. Because the games are unknown beforehand, the main problem lies in the detection and construction of useful features and heuristics for guiding search in the match.

One class of such features are distance features used in a variety of GGP agents (e.g., (Kuhlmann et al., 2006; Schiffel and Thielscher, 2007; Clune, 2007; Kaiser, 2007)). The way of detecting and constructing features in current game playing systems, however, suffers from a variety of disadvantages:

- Distance features require a prior recognition of board-like game elements. Current approaches formulate hypotheses about which element of the game rules describes a board and then either check these hypotheses in internal simulations of the game (e.g., (Kuhlmann et al., 2006; Schiffel and Thielscher, 2007; Kaiser, 2007)) or try to prove them (Schiffel and Thielscher, 2009a). Both approaches are expensive and can only detect boards if their description follows a certain syntactic pattern.

- Distance features are limited to Cartesian boardlike structures, that is, n-dimensional structures with totally ordered coordinates. Distances over general graphs are not considered.

- Distances are calculated using a predefined metric on the boards. Consequently, distance values obtained do not depend on the type of piece involved. For example, using a predefined metric the distance of a rook, king and pawn from $a 2$ to $c 2$ would appear equal while a human would identify the distance as 1,2 and $\infty$ (unreachable), respectively.

In this paper we will present a more general approach for the construction of distance features for general games. The underlying idea is to analyze the rules of game in order to find dependencies between the fluents of the game, i.e., between the atomic properties of the game states. Based on these dependencies, we define a distance function that computes an admissible estimate for the number of steps required to make a certain fluent true. This distance function 
can be used as a feature in search heuristics of GGP agents. In contrast to previous approaches, our approach does not depend on syntactic patterns and involves no internal simulation or detection of any predefined game elements. Moreover, it is not limited to board-like structures but can be used for every fluent of a game.

The remainder of this paper is structured as follows: In the next section we give an introduction to the Game Description Language (GDL), which is used to describe general games. Furthermore, we briefly present the methods currently applied for distance feature detection and distance estimation in the field of General Game Playing. In Section 3 we introduce the theoretical basis for this work, so called fluent graphs, and show how to use them to derive distances from states to fluents. We proceed in Section 4 by showing how fluent graphs can be constructed from a game description and demonstrate their application in Section 5. Finally, we conduct experiments in Section 6 to show the benefit and generality of our approach and discuss and summarize the results in Section 9.

\section{Preliminaries}

The language used for describing the rules of general games is the Game Description Language (Love et al., 2008) (GDL). GDL is an extension of Datalog with functions, equality, some syntactical restrictions to preserve finiteness, and some predefined keywords.

The following is a partial encoding of a Tic-TacToe game in GDL. In this paper we use Prolog syntax where words starting with upper-case letters stand for variables and the remaining words are constants.

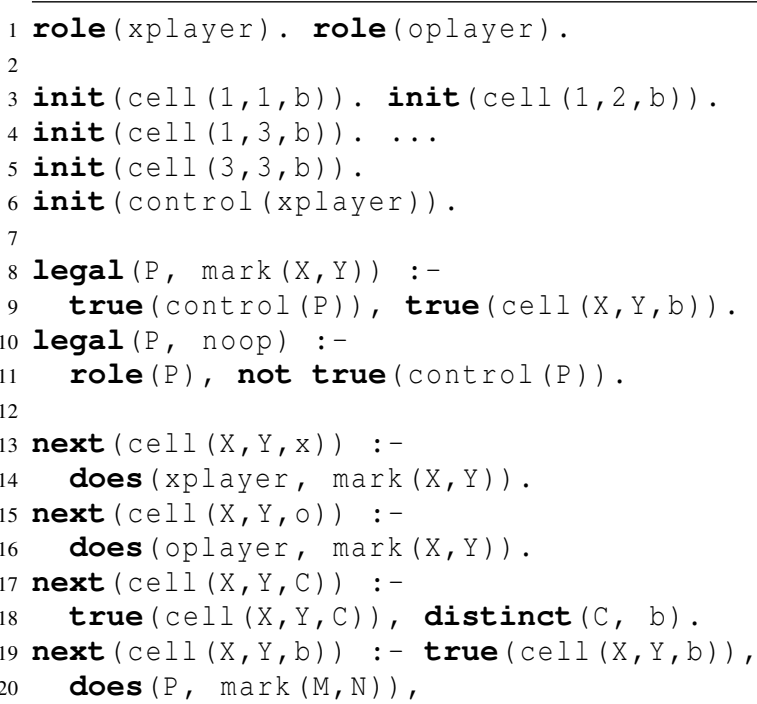

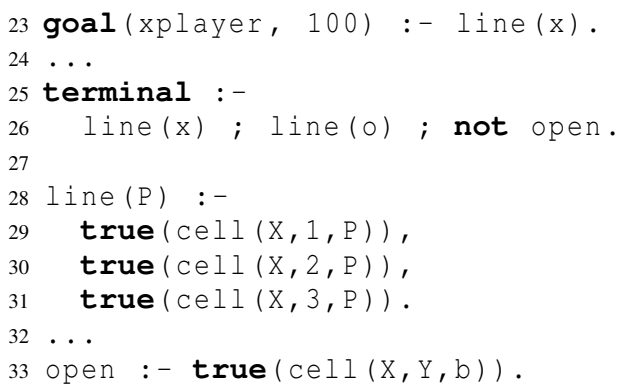

23 goal (xplayer, 100) :- line (x).

24 ...

terminal :-

line $(x)$; line (o); not open.

line (P) :-

true $(\operatorname{cell}(X, 1, P))$

true $(\operatorname{cell}(X, 2, P))$

true $(\operatorname{cell}(X, 3, P))$.

$\ldots$

open :- true $(\operatorname{cell}(X, Y, b))$.

21

22

The first line declares the roles of the game. The unary predicate init defines the properties that are true in the initial state. Lines 8-11 define the legal moves of the game with the help of the keyword legal. For example, mark $(X, Y)$ is a legal move for role $P$ if control $(P)$ is true in the current state (i.e., it's P's turn) and the cell $X, Y$ is blank $(\operatorname{cell}(X, Y, b))$. The rules for predicate next define the properties that hold in the successor state, e.g., $\operatorname{cell}(\mathrm{M}, \mathrm{N}, \mathrm{x})$ holds if xplayer marked the cell $M, N$ and $\operatorname{cell}(M, N, b)$ does not change if some cell different from $\mathrm{M}, \mathrm{N}$ was marked $^{1}$. Lines 23 to 26 define the rewards of the players and the condition for terminal states. The rules for both contain auxiliary predicates line (P) and open which encode the concept of a line-of-three and the existence of a blank cell, respectively.

We will refer to the arguments of the GDL keywords init, true and next as fluents. In the above example, there are two different types of fluents, control (X) with $\mathrm{x} \in$ \{xplayer, oplayer and $\operatorname{cell}(X, Y, Z)$ with $X, Y \in\{1,2,3\}$ and $Z \in$ $\{b, x, o\}$.

In (Schiffel and Thielscher, 2009b), we defined a formal semantics of a game described in GDL as a state transition system:

Definition 1 (Game). Let $\Sigma$ be a set of ground terms and $2^{\Sigma}$ denote the set of finite subsets of $\Sigma$. A game over this set of ground terms $\Sigma$ is a state transition system $\Gamma=\left(R, s_{0}, T, l, u, g\right)$ over sets of states $\mathcal{S} \subseteq 2^{\Sigma}$ and actions $\mathcal{A} \subseteq \Sigma$ with

- $R \subseteq \Sigma$, a finite set of roles;

- $s_{0} \in S$, the initial state of the game;

- $T \subseteq \mathcal{S}$, the set of terminal states;

- $l: R \times \mathcal{A} \times \mathcal{S}$, the legality relation;

- $u:(R \mapsto \mathcal{A}) \times S \rightarrow S$, the transition or update function; and

- $g: R \times \mathcal{S} \mapsto \mathbb{N}$, the reward or goal function.

\footnotetext{
${ }^{1}$ The special predicate $\operatorname{distinct}(X, Y)$ holds if the terms $X$ and $Y$ are syntactically different.
} 
This formal semantics is based on a set of ground terms $\Sigma$. This set is the set of all ground terms over the signature of the game description. Hence, fluents, actions and roles of the game are ground terms in $\Sigma$. States are finite sets of fluents, i.e., finite subsets of $\Sigma$. The connection between a game description $D$ and the game $\Gamma$ it describes is established using the standard model of the logic program $D$. For example, the update function $u$ is defined as

$$
u(A, s)=\left\{f \in \Sigma: D \cup s^{\text {true }} \cup A^{\text {does }} \models \operatorname{next}(f)\right\}
$$

where $s^{\text {true }}$ and $A^{\text {does }}$ are suitable encodings of the state $s$ and the joint action $A$ of all players as a logic program. Thus, the successor state $u(A, s)$ is the set of all ground terms (fluents) $f$ such that next $(f)$ is entailed by the game description $D$ together with the state $s$ and the joint move $A$. For a complete definition for all components of the game $\Gamma$ we refer to (Schiffel and Thielscher, 2009b).

\section{Fluent Graphs}

Our goal is to obtain knowledge on how fluents evolve over time. We start by building a fluent graph that contains all the fluents of a game as nodes. Then we add directed edges $\left(f_{i}, f\right)$ if at least one of the predecessor fluents $f_{i}$ must hold in the current state for the fluent $f$ to hold in the successor state. Figure 1 shows a partial fluent graph for Tic-Tac-Toe that relates the fluents $\operatorname{cell}(3,1, z)$ for $z \in\{b, x, 0\}$.

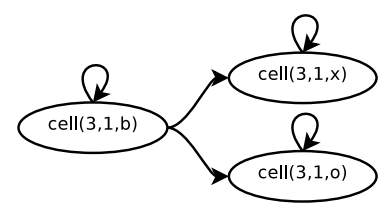

Figure 1: Partial fluent graph for Tic-Tac-Toe.

For cell $(3,1)$ to be blank it had to be blank before. For a cell to contain an $\mathrm{x}$ (or an $\circ$ ) in the successor state there are two possible preconditions. Either, it contained an $\mathrm{x}$ (or $\mathrm{\circ}$ ) before or it was blank.

Using this graph, we can conclude that, e.g., a transition from $\operatorname{cell}(3,1, b)$ to $\operatorname{cell}(3,1, x)$ is possible within one step while a transition from $\operatorname{cell}(3,1,0)$ to $\operatorname{cell}(3,1, x)$ is impossible.

To build on this information, we formally define a fluent graph as follows:

Definition 2 (Fluent Graph). Let $\Gamma$ be a game over ground terms $\Sigma$. A graph $G=(V, E)$ is called a fluent graph for $\Gamma$ iff

- $V=\Sigma \cup\{\emptyset\}$ and
- for all fluents $f \in \Sigma$, two valid states $s$ and $s^{\prime}$

$$
\begin{aligned}
& \left(s^{\prime} \text { is a successor of } s\right) \wedge f^{\prime} \in s^{\prime} \\
& \quad \Rightarrow(\exists f)\left(f, f^{\prime}\right) \in E \wedge(f \in s \cup\{\emptyset\})
\end{aligned}
$$

In this definition we add an additional node $\emptyset$ to the graph and allow $\emptyset$ to occur as the source of edges. The reason is that there can be fluents in the game that do not have any preconditions, for example the fluent $g$ with the following next rule: next $(g)$ :- distinct $(a, b)$. On the other hand, there might be fluents that cannot occur in any state, because the body of the corresponding next rule is unsatisfiable, for example: next $(h)$ :- distinct $(a, a)$. We distinguish between fluents that have no precondition (such as $g$ ) and fluents that are unreachable (such as $h$ ) by connecting the former to the node $\emptyset$ while unreachable fluents have no edge in the fluent graph.

Note that the definition covers only some of the necessary preconditions for fluents, therefore fluent graphs are not unique as Figure 2 shows. We will address this problem later.

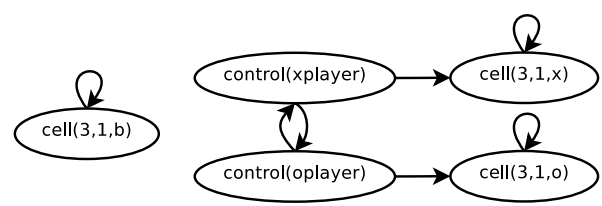

Figure 2: Alternative partial fluent graph for Tic-Tac-Toe.

We can now define a distance function $\Delta\left(s, f^{\prime}\right)$ between the current state $s$ and a state in which fluent $f^{\prime}$ holds as follows:

Definition 3 (Distance Function). Let $\Delta_{G}\left(f, f^{\prime}\right)$ be the length of the shortest path from node $f$ to node $f^{\prime}$ in the fluent graph $G$ or $\infty$ if there is no such path. Then

$$
\Delta\left(s, f^{\prime}\right)=\min _{f \in s \cup\{\emptyset\}} \Delta_{G}\left(f, f^{\prime}\right)
$$

That means, we compute the distance $\Delta\left(s, f^{\prime}\right)$ as the shortest path in the fluent graph from any fluent in $s$ to $f^{\prime}$.

Intuitively, each edge $\left(f, f^{\prime}\right)$ in the fluent graph corresponds to a state transition of the game from a state in which $f$ holds to a state in which $f^{\prime}$ holds. Thus, the length of a path from $f$ to $f^{\prime}$ in the fluent graph corresponds to the number of steps in the game between a state containing $f$ to a state containing $f^{\prime}$. Of course, the fluent graph is an abstraction of the actual game: many preconditions for the state transitions are ignored. As a consequence, the distance $\Delta\left(s, f^{\prime}\right)$ that we compute in this way is a lower bound on the actual number of steps it takes to go from $s$ to a state in which $f^{\prime}$ holds. Therefore the distance 
$\Delta\left(s, f^{\prime}\right)$ is an admissible heuristic for reaching $f^{\prime}$ from a state $s$.

Theorem 1 (Admissible Distance). Let

- $\Gamma=\left(R, s_{0}, T, l, u, g\right)$ be a game with ground terms $\Sigma$ and states $\mathcal{S}$,

- $s_{1} \in \mathcal{S}$ be a state of $\Gamma$,

- $f \in \Sigma$ be a fluent of $\Gamma$, and

- $G=(V, E)$ be a fluent graph for $\Gamma$.

Furthermore, let $s_{1} \mapsto s_{2} \mapsto \ldots \mapsto s_{m+1}$ denote a legal sequence of states of $\Gamma$, that is, for all $i$ with $0<i \leq m$ there is a joint action $A_{i}$, such that:

$$
s_{i+1}=u\left(A_{i}, s_{i}\right) \wedge(\forall r \in R) l\left(r, A_{i}(r), s\right)
$$

If $\Delta\left(s_{1}, f\right)=n$, then there is no legal sequence of states $s_{1} \mapsto \ldots \mapsto s_{m+1}$ with $f \in s_{m+1}$ and $m<n$.

Proof. We prove the theorem by contradiction. Assume that $\Delta\left(s_{1}, f\right)=n$ and there is a legal sequence of states $s_{1} \mapsto \ldots \mapsto s_{m+1}$ with $f \in s_{m+1}$ and $m<n$. By Definition 2, for every two consecutive states $s_{i}$, $s_{i+1}$ of the sequence $s_{1} \mapsto \ldots \mapsto s_{m+1}$ and for every $f_{i+1} \in s_{i+1}$ there is an edge $\left(f_{i}, f_{i+1}\right) \in E$ such that $f_{i} \in s_{i}$ or $f_{i}=\emptyset$. Therefore, there is a path $f_{j}, \ldots, f_{m}, f_{m+1}$ in $G$ with $1 \leq j \leq m$ and the following properties:

- $f_{i} \in s_{i}$ for all $i=j, \ldots, m+1$,

- $f_{m+1}=f$, and

- either $f_{j} \in s_{1}$ (e.g., if $j=1$ ) or $f_{j}=\emptyset$.

Thus, the path $f_{j}, \ldots, f_{m}, f_{m+1}$ has a length of at most $m$. Consequently, $\Delta\left(s_{1}, f\right) \leq m$ because $f_{j} \in s_{1} \cup\{\emptyset\}$ and $f_{m+1}=f$. However, $\Delta\left(s_{1}, f\right) \leq m$ together with $m<n$ contradicts $\Delta\left(s_{1}, f\right)=n$.

\section{Constructing Fluent Graphs from Rules}

We propose an algorithm to construct a fluent graph based on the rules of the game. The transitions of a state $s$ to its successor state $s^{\prime}$ are encoded fluent-wise via the next rules. Consequently, for each $f^{\prime} \in s^{\prime}$ there must be at least one rule with the head next $\left(f^{\prime}\right)$. All fluents occurring in the body of these rules are possible sources for an edge to $f^{\prime}$ in the fluent graph.

For each ground fluent $f^{\prime}$ of the game:

1. Construct a ground disjunctive normal form $\phi$ of next $\left(f^{\prime}\right)$, i.e., a formula $\phi$ such that next $\left(f^{\prime}\right) \supset \phi$.

2. For every disjunct $\psi$ in $\phi$ :

- Pick one literal true $(f)$ from $\psi$ or set $f=\emptyset$ if there is none.

- Add the edge $\left(f, f^{\prime}\right)$ to the fluent graph.
Note, that we only select one literal from each disjunct in $\phi$. Since, the distance function $\Delta\left(s, f^{\prime}\right)$ obtained from the fluent graph is admissible, the goal is to construct a fluent graph that increases the lengths of the shortest paths between the fluents as much as possible. Therefore, the fluent graph should contain as few edges as possible. In general the complete fluent graph (i.e., the graph where every fluent is connected to every other fluent) is the least informative because the maximal distance obtained from this graph is 1 .

The algorithm outline still leaves some open issues:

1. How do we construct a ground formula $\phi$ that is the disjunctive normal form of next $\left(f^{\prime}\right)$ ?

2. Which literal true $(f)$ do we select if there is more than one? Or, in other words, which precondition $f^{\prime}$ of $f$ do we select?

We will discuss both issues in the following sections.

\subsection{Constructing a DNF of next $\left(\mathbf{f}^{\prime}\right)$}

A formula $\phi$ in DNF is a set of formulas $\left\{\psi_{1}, \ldots, \psi_{n}\right\}$ connected by disjunctions such that each formula $\psi_{i}$ is a set of literals connected by conjunctions. We propose the algorithm in Figure 1 to construct $\phi$ such that $\operatorname{next}\left(f^{\prime}\right) \supset \phi$.

The algorithm starts with $\phi=\operatorname{next}\left(f^{\prime}\right)$. Then, it selects a positive literal $l$ in $\phi$ and unrolls this literal, that is, it replaces $l$ with the bodies of all rules $h:-b \in D$ whose head $h$ is unifiable with $l$ with a most general unifier $\sigma$ (lines 9,10$)$. The replacement is repeated until all predicates that are left are either of the form true $(t)$, distinct $\left(t_{1}, t_{2}\right)$ or recursively defined. Recursively defined predicates are not unrolled to ensure termination of the algorithm. Finally, we transform $\phi$ into disjunctive normal form and replace each disjunct $\psi_{i}$ of $\phi$ by a disjunction of all of its ground instances in order to get a ground formula $\phi$.

Note that in line 4 , we replace every occurrence of does with legal to also include the preconditions of the actions that are executed in $\phi$. As a consequence the resulting formula $\phi$ is not equivalent to next $\left(f^{\prime}\right)$. However, next $\left(f^{\prime}\right) \supset \phi$, under the assumption that only legal moves can be executed, i.e., $\operatorname{does}(r, a) \supset \operatorname{legal}(r, a)$. This is sufficient for constructing a fluent graph from $\phi$.

Note, that we do not select negative literals for unrolling. The algorithm could be easily adapted to also unroll negative literals. However, in the games we encountered so far, doing so does not improve the obtained fluent graphs but complicates the algorithm and increases the size of the created $\phi$. Unrolling negative 


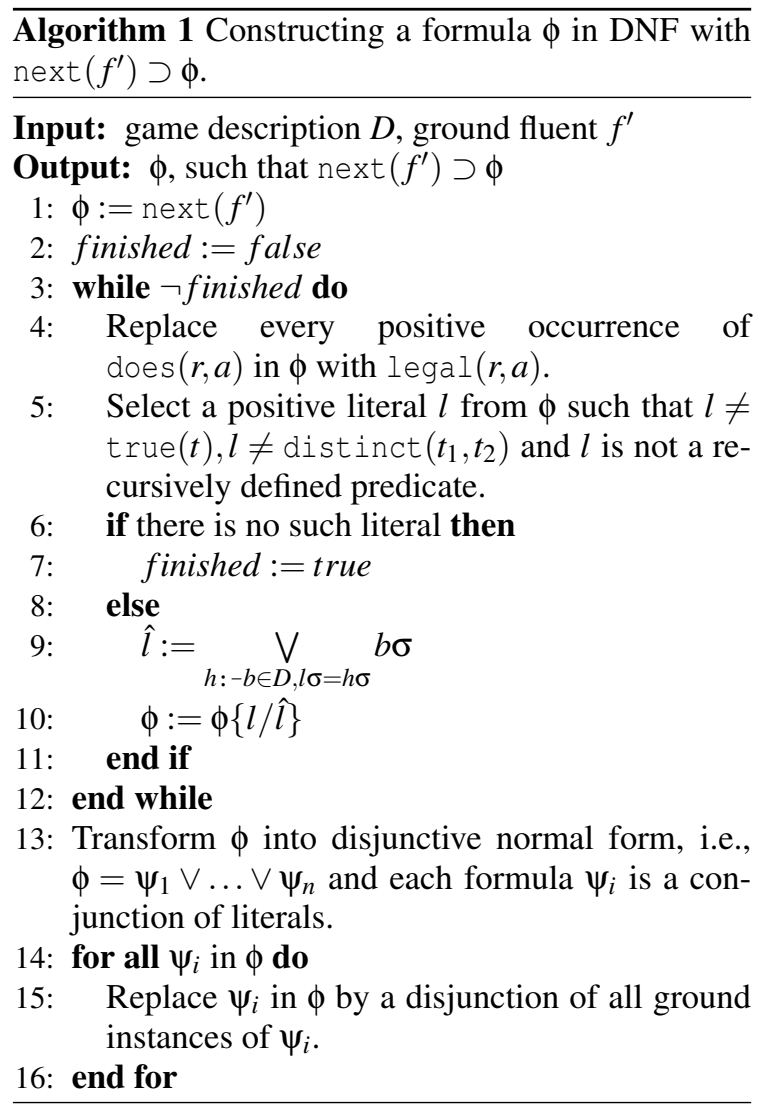

literals will mainly add negative preconditions to $\phi$. However, negative preconditions are not used for the fluent graph because a fluent graph only contains positive preconditions of fluents as edges, according to Definition 2.

\subsection{Selecting Preconditions for the Fluent Graph}

If there are several literals of the form $\operatorname{true}(f)$ in a disjunct $\psi$ of the formula $\phi$ constructed above, we have to select one of them as source of the edge in the fluent graph. As already mentioned, the distance $\Delta(s, f)$ computed with the help of the fluent graph is a lower bound on the actual number of steps needed. To obtain a good lower bound, that is one that is as large as possible, the paths between nodes in the fluent graph should be as long as possible. Selecting the best fluent graph, i.e., the one which maximizes the distances, is impossible. Which fluent graph is the best one depends on the states we encounter when playing the game, but we do not know these states beforehand. In order to generate a fluent graph that provides good distance estimates, we use several heuristics when we select literals from disjuncts in the DNF of next $\left(f^{\prime}\right)$ :
First, we only add new edges if necessary. That means, whenever there is a literal true $(f)$ in a disjunct $\psi$ such that the edge $\left(f, f^{\prime}\right)$ already exists in the fluent graph, we select this literal true $(f)$. The rationale of this heuristic is that paths in the fluent graph are longer on average if there are fewer connections between the nodes.

Second, we prefer a literal true $(f)$ over true $(g)$ if $f$ is more similar to $f^{\prime}$ than $g$ is to $f^{\prime}$, that is $\operatorname{sim}\left(f, f^{\prime}\right)>\operatorname{sim}\left(g, f^{\prime}\right)$.

We define the similarity $\operatorname{sim}\left(t, t^{\prime}\right)$ recursively over ground terms $t, t^{\prime}$ :

$\operatorname{sim}\left(t, t^{\prime}\right)= \begin{cases}1 & t, t^{\prime} \text { have arity } 0 \text { and } t=t^{\prime} \\ \sum_{i} \operatorname{sim}\left(t_{i}, t_{i}^{\prime}\right) & t=f\left(t_{1}, \ldots, t_{n}\right) \text { and } \\ & t^{\prime}=f\left(t_{1}^{\prime}, \ldots, t_{n}^{\prime}\right) \\ 0 & \text { else }\end{cases}$

In human made game descriptions, similar fluents typically have strong connections. For example, in Tic-Tac-Toe cell $(3,1, x)$ is more related to $\operatorname{cell}(3,1, b)$ than to cell $(b, 3, x)$. By using similar fluents when adding new edges to the fluent graph, we have a better chance of finding the same fluent again in a different disjunct of $\phi$. Thus we maximize the chance of reusing edges.

\section{Applying Distance Features}

For using the distance function in our evaluation function, we define the normalized distance $\delta(s, f)$.

$$
\delta(s, f)=\frac{\Delta(s, f)}{\Delta_{\max }(f)}
$$

The value $\Delta_{\max }(f)$ is the longest distance $\Delta_{G}(g, f)$ from any fluent $g$ to $f$, i.e.,

$$
\Delta_{\max }(f) \stackrel{\text { def }}{=} \max _{g} \Delta_{G}(g, f)
$$

where $\Delta_{G}(g, f)$ denotes the length of the shortest path from $g$ to $f$ in the fluent graph $G$.

Thus, $\Delta_{\max }(f)$ is the longest possible distance $\Delta(s, f)$ that is not infinite. The normalized distance $\delta(s, f)$ will be infinite if $\Delta(s, f)=\infty$, i.e., there is no path from any fluent in $s$ to $f$ in the fluent graph. In all other cases it holds that $0 \leq \delta(s, f) \leq 1$.

Note, that the construction of the fluent graph and computing the shortest paths between all fluents, i.e., the distance function $\Delta_{G}$, need only be done once for a game. Thus, while construction of the fluent graph is more expensive for complex games, the cost of computing the distance feature $\delta(s, f)$ (or $\Delta(s, f)$ ) only depends (linearly) on the size of the state $s$. 


\subsection{Using Distance Features in an Evaluation Function}

To demonstrate the application of the distance measure presented, we use a simplified version of the evaluation function of Fluxplayer (Schiffel and Thielscher, 2007) implemented in Prolog. It takes the ground DNF of the goal rules as first argument, the current state as second argument and returns the fuzzy evaluation of the DNF on that state as a result.

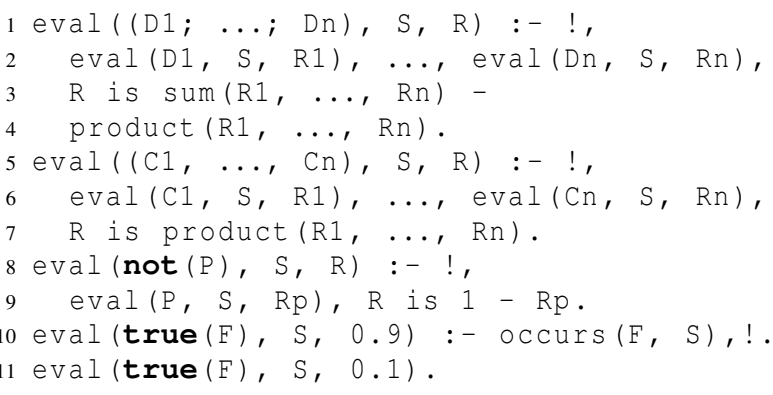

Disjunctions are transformed to probabilistic sums, conjunctions to products, and true statements are evaluated to values in the interval $[0,1]$, basically resembling a recursive fuzzy logic evaluation using the product t-norm and the corresponding probabilistic sum t-conorm. The state value increases with each conjunct and disjunct fulfilled.

We compare this basic evaluation to a second function that employs our relative distance measure, encoded as predicate delta. We obtain this distancebased evaluation function by substituting line 11 of the previous program by the following four lines:

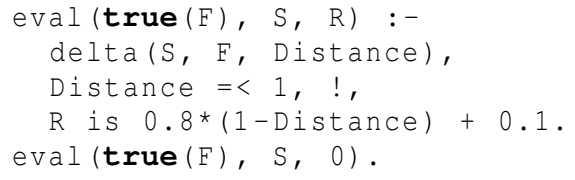

Here, we evaluate a fluent that does not occur in the current state to a value in $[0.1,0.9]$ and, in case the relative distance is infinite, to 0 since this means that the fluent cannot hold anymore.

\subsection{Tic-Tac-Toe}

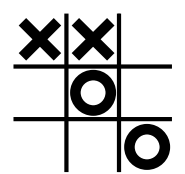

state $\mathrm{s}_{1}$

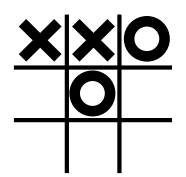

state $\mathrm{s}_{2}$
Figure 3: Two states of the Tic-Tac-Toe. The first row is still open in state $s_{1}$ but blocked in state $s_{2}$.
Although on first sight Tic-Tac-Toe contains no relevant distance information, we can still take advantage of our distance function. Consider the two states as shown in Figure 3. In state $s_{1}$ the first row consists of two cells marked with an $\mathrm{x}$ and a blank cell. In state $s_{2}$ the first row contains two xs and one cell marked with an $\circ$. State $s_{1}$ has a higher state value than $s_{2}$ for xplayer since in $s_{1}$ xplayer has a threat of completing a line in contrast to $s_{2}$. The corresponding goal condition for xplayer completing the first row is:

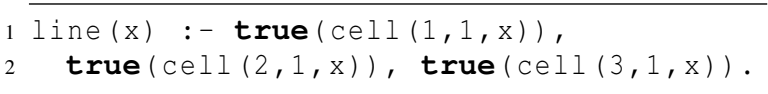

When evaluating the body of this condition using our standard fuzzy evaluation, we see that it cannot distinguish between $s_{1}$ and $s_{2}$ because both have two markers in place and one missing for completing the line for xplayer. Therefore it evaluates both states to $1 * 1 * 0.1=0.1$.

However, the distance-based function evaluates true $(\operatorname{cell}(3,1, b))$ of $s_{1}$ to 0.1 and true $(\operatorname{cell}(3,1,0))$ of $s_{2}$ to 0 . Therefore, it can distinguish between both states, returning $R=0.1$ for $\mathrm{S}=s_{1}$ and $\mathrm{R}=0$ for $\mathrm{S}=s_{2}$.

\subsection{Breakthrough}

The second game is Breakthrough, again a two-player game played on a chessboard. Like in chess, the first two ranks contain only white pieces and the last two only black pieces. The pieces of the game are only pawns that move and capture in the same way as pawns in chess, but without the initial double advance. Whoever reaches the opposite side of the board first wins. ${ }^{2}$ Figure 4 shows the initial position for Breakthrough. The arrows indicate the possible moves, a pawn can make.

The goal condition for the player black states that black wins if there is a cell with the coordinates $x, 1$ and the content black, such that $\mathrm{X}$ is an index (a number from 1 to 8 according to the rules of index):

$1 \overline{\text { goal (black, 100): }:-}$
2 index (X), true (cellholds (X, 1, black)).

Grounding this rule yields

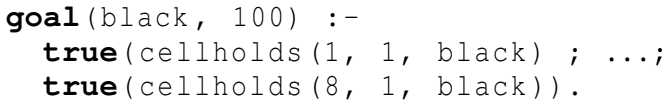

We omitted the index predicate since it is true for all 8 ground instances.

${ }^{2}$ The complete rules for Breakthrough
as well as Tic-Tac-Toe can be found under
http://ggpserver.general-game-playing.de/


The standard evaluation function cannot distinguish any of the states in which the goal is not reached because true (cellholds ( $X, 1$, black)) is false in all of these states for any instance of $x$.

The distance-based evaluation function is based on the fluent graph depicted in Figure 5.

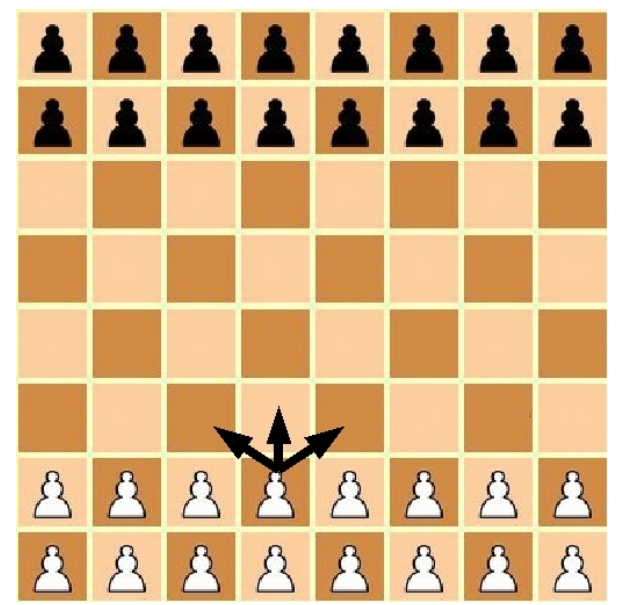

Figure 4: Initial position in Breakthrough and the move options of a pawn.

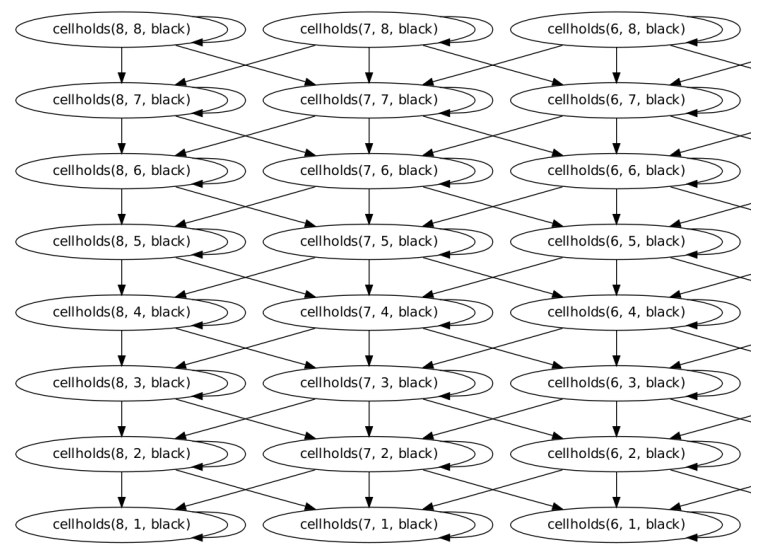

Figure 5: A partial fluent graph for Breakthrough, role black.

Obviously it captures the way pawns move in chess. Therefore evaluations of atoms of the form true (cellholds (X, Y, black)) have now 9 possible values (for distances 0 to 7 and $\infty$ ) instead of 2 (true and false). Hence, states where black pawns are nearer to one of the cells $(1,8), \ldots,(8,8)$ are preferred.

Moreover, the fluent graph, and thus the distance function, contains the information that some locations are only reachable from certain other locations. Together with our evaluation function this leads to what could be called "strategic positioning": states with pawns on the side of the board are worth less than those with pawns in the center. This is due to the fact that a pawn in the center may reach more of the 8 possible destinations than a pawn on the side.

\section{Evaluation}

For evaluation, we implemented our distance function and equipped the agent system Fluxplayer (Schiffel and Thielscher, 2007) with it. We then set up this version of Fluxplayer ("flux_distance") against its version without the new distance function ("flux_basic"). We used the version of Fluxplayer that came in 4th in the 2010 championship. Since flux_basic is already endowed with a distance heuristic, the evaluation is comparable to a competition setting of two competing heuristics using distance features.

We chose 19 games for comparison in which we conducted 100 matches on average. Figure 6 shows the results.

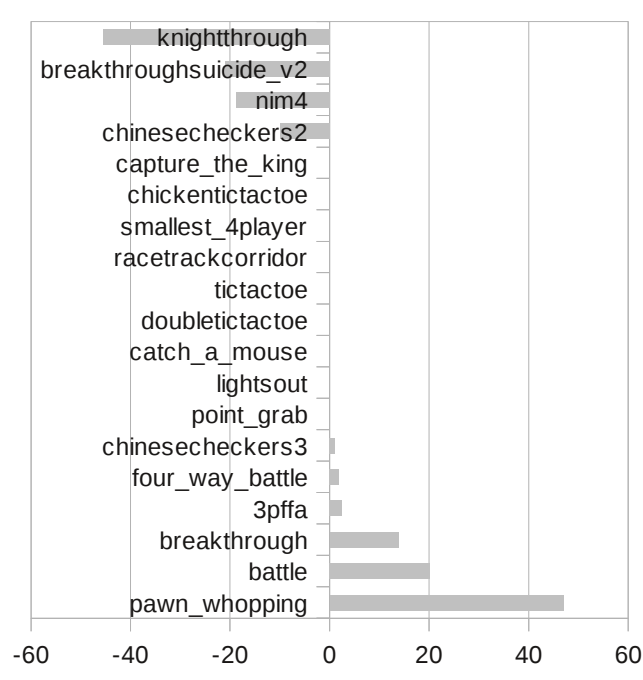

Figure 6: Advantage in Win Rate of flux_distance

The values indicate the difference in win rate, e.g., a value of +10 indicates that flux_distance won $55 \%$ of the games against flux_basic winning $45 \%$. Obviously the proposed heuristics produces results comparable to the flux_basic heuristics, with both having advantages in some games. This has several reasons: Most importantly, our proposed heuristic, in the way it is implemented now, is more expensive than the distance estimation used in flux_basic. Therefore the evaluation of a state takes longer and the search tree can not be explored as deeply as with cheaper heuristics. This accounts for three of the four underperforming games. For example in nim4, the flux_basic 
distance estimation provides essentially the same results as our new approach, just much cheaper. In chinesecheckers 2 and knightthrough, the new distance function slows down the search more than its better accuracy can compensate.

On the other hand, flux_distance performs better in complicated games. There the higher accuracy of the heuristics typically outweighs the disadvantage of the heuristics being slower.

Interestingly the higher accuracy of the new distance heuristics is the reason for flux_distance losing in breakthrough_suicide. The game is exactly the same as breakthrough, however, the player to reach the other side of the board first does not win but loses.

The heuristics of both flux_basic and flux_distance are not good for this game since both are based the minimum number of moves necessary to reach the goal while the optimal heuristic would depend on the maximum number of moves available to avoid losing. However, since flux_distance is more accurate, flux_distance selects even worse moves that flux_basic. Specifically, flux distance tries to maximize (a much more accurate) minimal distance to the other side of the board, thereby allowing the opponent to capture its advanced pawns. This behavior, however, results in a smaller maximal number of moves until the game ends and forces to advance the few remaining pawns quickly. Thus, the problem in this game is not the distance estimate but the fact that the heuristic is not suitable for the game.

Finally, in some of the games no changes were found since both distance estimates performed equally well. However, rather specific heuristics and analysis methods of flux_basic could be replaced by our new general approach. For example, the original Fluxplayer contains a special method to detect when a fluent is unreachable, while this information is automatically included in our distance estimate.

Apart from the above results, we intended to use more games for evaluation, however, we found that the fluent graph construction takes too much time in some games where the next rules are complex. We discuss these issues in Section 8.

\section{Related Work}

Distance features are part of classical agent programming for games like chess and checkers in order to measure, e.g., the distance of a pawn to the promotion rank. A more general detection mechanism was first employed in Metagamer (Pell, 1993) where the features "promote-distance" and "arrivaldistance" represented a value indirectly proportional to the distance of a piece to its arrival or promotion square. However, due to the restriction on symmetric chess-like games, boards and their representation were predefined and thus predefined features could be applied as soon as some promotion or arrival condition was found in the game description.

Currently, a number of GGP agent systems apply distance features in different forms. UTexas (Kuhlmann et al., 2006) identifies order relations syntactically and tries to find $2 \mathrm{~d}$-boards with coordinates ordered by these relations. Properties of the content of these cells, such as minimal/maximal $x$ - and $y$-coordinates or pair-wise Manhattan distances are then assumed as candidate features and may be used in the evaluation function. Fluxplayer (Schiffel and Thielscher, 2007) generalizes the detection mechanism using semantic properties of order relations and extends board recognition to arbitrarily defined $n$-dimensional boards.

Another approach is pursued by Cluneplayer (Clune, 2007) who tries to impose a symbol distance interpretation on expressions found in the game description. Symbol distances, however, are again calculated using Manhattan distances on ordered arguments of board-like fluents, eventually resulting in a similar distance estimate as UTexas and Fluxplayer.

Although not explained in detail, Ogre (Kaiser, 2007) also employs two features that measure the distance from the initial position and the distance to a target position. Again, Ogre relies on syntactic detection of order relations and seems to employ a board centered metrics, ignoring the piece type.

All of these approaches rely on the identification of certain fixed structures in the game (such as game boards) but can not be used for fluents that do not belong to such a structure. Furthermore, they make assumptions about the distances on these structures (usually Manhattan distance) that are not necessarily connected to the game dynamics, e.g., how different pieces move on a board.

In domain independent planning, distance heuristics are used successfully, e.g., in HSP (Bonet and Geffner, 2001) and FF (Hoffmann and Nebel, 2001). The heuristics $h(s)$ used in these systems is an approximation of the plan length of a solution in a relaxed problem, where negative effects of actions are ignored. This heuristics is known as delete list relaxation. While on first glance this may seems very similar to our approach, several differences exist:

- The underlying languages, GDL for general game playing and PDDL for planning, are different. A translation of GDL to PDDL is expensive in many games (Kissmann and Edelkamp, 2010). Thus, 
directly applying planning systems is not often not feasible.

- The delete list relaxation considers all (positive) preconditions of a fluent, while we only use one precondition. This enables us to precompute the distance between the fluents of a game.

- While goal conditions of most planning problems are simple conjunctions, goals in the general games can be very complex (e.g., checkmate in chess). Additionally, the plan length is usually not a good heuristics, given that only the own actions and not those of the opponents can be controlled. Thus, distance estimates in GGP are usually not used as the only heuristics but only as a feature in a more complex evaluation function. As a consequence, computing distance features must be relatively cheap.

- Computing the plan length of the relaxed planning problem is NP-hard, and even the approximations used in HSP or FF that are not NP-hard require to search the state space of the relaxed problem. On the other hand, computing distance estimates with our solution is relatively cheap. The distances $\Delta_{G}(f, g)$ between all fluents $f$ and $g$ in the fluent graph can be precomputed once for a game. Then, computing the distance $\Delta\left(s, f^{\prime}\right)$ (see Definition 3 ) is linear in the size of the state $s$, i.e., linear in the number of fluents in the state.

\section{Future Work}

The main problem of the approach is its computational cost for constructing the fluent graph. The most expensive steps of the fluent graph construction are grounding of the DNF formulas $\phi$ and processing the resulting large formulas to select edges for the fluent graph. For many complex games, these steps cause either out-of-memory or time-out errors. Thus, an important line of future work is to reduce the size of formulas before the grounding step without losing relevant information.

One way to reduce the size of $\phi$ is a more selective expansion of predicates (line 5) in Algorithm 1. Developing heuristics for this selection of predicates is one of the goals for future research.

In addition, we are working on a way to construct fluent graphs from non-ground representations of the preconditions of a fluent to skip the grounding step completely. For example, the partial fluent graph in Figure 1 is identical to the fluent graphs for the other 8 cells of the Tic-Tac-Toe board. The fluent graphs for all 9 cells are obtained from the same rules for next ( $\operatorname{cell}\left(X, Y,{ }_{-}\right)$, just with different instances of the variables $X$ and $Y$. By not instantiating $X$ and $Y$, the generated DNF is exponentially smaller while still containing the same information.

The quality of the distance estimates depends mainly on the selection of preconditions. At the moment, the heuristics we use for this selection are intuitive but have no thorough theoretic or empiric foundation. In future, we want to investigate how these heuristics can be improved.

Furthermore, we intend to enhance the approach to use fluent graphs for generalizations of other types of features, such as, piece mobility and strategic positions.

\section{Summary}

We have presented a general method of deriving distance estimates in General Game Playing. To obtain such a distance estimate, we introduced fluent graphs, proposed an algorithm to construct them from the game rules and demonstrated the transformation from fluent graph distance to a distance feature.

Unlike previous distance estimations, our approach does not rely on syntactic patterns or internal simulations. Moreover, it preserves piece-dependent move patterns and produces an admissible distance heuristic.

We showed on an example how these distance features can be used in a state evaluation function. We gave two examples on how distance estimates can improve the state evaluation and evaluated our distance against Fluxplayer in its most recent version.

Certain shortcomings should be addressed to improve the efficiency of fluent graph construction and the quality of the obtained distance function. Despite these shortcomings, we found that a state evaluation function using the new distance estimates can compete with a state-of-the-art system.

\section{REFERENCES}

Bonet, B. and Geffner, H. (2001). Planning as heuristic search. Artificial Intelligence, 129(1-2):5-33.

Clune, J. (2007). Heuristic evaluation functions for general game playing. In $A A A I$, Vancouver. AAAI Press.

Hoffmann, J. and Nebel, B. (2001). The FF planning system: Fast plan generation through heuristic search. JAIR, 14:253-302.

Kaiser, D. M. (2007). Automatic feature extraction for autonomous general game playing agents. In Proceedings of the Sixth Intl. Joint Conf. on Autonomous Agents and Multiagent Systems. 
Kissmann, P. and Edelkamp, S. (2010). Instantiating general games using prolog or dependency graphs. In Dillmann, R., Beyerer, J., Hanebeck, U. D., and Schultz, T., editors, KI, volume 6359 of Lecture Notes in Computer Science, pages 255-262. Springer.

Kuhlmann, G., Dresner, K., and Stone, P. (2006). Automatic Heuristic Construction in a Complete General Game Player. In Proceedings of the Twenty-First National Conference on Artificial Intelligence, pages 1457-62, Boston, Massachusetts, USA. AAAI Press.

Love, N., Hinrichs, T., Haley, D., Schkufza, E., and Genesereth, M. (2008). General game playing: Game description language specification. Technical Report March 4, Stanford University. The most recent version should be available at http://games.stanford.edu/.

Pell, B. (1993). Strategy generation and evaluation for meta-game playing. $\mathrm{PhD}$ thesis, University of Cambridge.

Schiffel, S. and Thielscher, M. (2007). Fluxplayer: A successful general game player. In Proceedings of the National Conference on Artificial Intelligence, pages 1191-1196, Vancouver. AAAI Press.

Schiffel, S. and Thielscher, M. (2009a). Automated theorem proving for general game playing. In Proceedings of the International Joint Conference on Artificial Intelligence (IJCAI).

Schiffel, S. and Thielscher, M. (2009b). A multiagent semantics for the game description language. In Filipe, J., Fred, A., and Sharp, B., editors, International Conference on Agents and Artificial Intelligence (ICAART), Porto. Springer. 